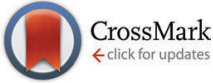

Cite this: Phys. Chem. Chem. Phys., 2015, 17, 6786

Received 2nd December 2014, Accepted 30th January 2015

DOI: $10.1039 / c 4 c p 05590 j$

www.rsc.org/pccp

\title{
Durable superhydrophilic/phobic surfaces based on green patina with corrosion resistance $\dagger$
}

\author{
Handong Cho, ${ }^{a}$ Jeongwon Lee, ${ }^{a}$ Sangmin Lee ${ }^{{ }^{b}}$ and Woonbong Hwang ${ }^{\star^{a}}$
}

\begin{abstract}
Special wetting surfaces with superhydrophilicity or superhydrophobicity have attracted great interest because of their potential for practical applications. However, since the special wetting surface may be used in a severe environment, including polluted air and seawater, it is necessary to develop a durable special wetting surface with excellent corrosion-resistance. Here, we report a new strategy for robust superhydrophilic or superhydrophobic green patina surfaces on copper substrates with superior corrosion resistance and adhesion strength, which have great potential for treating marine pollution. The as-prepared surfaces exhibited superhydrophilicity with underwater superoleophobicity or superoleophilicity with under-oil superhydrophobicity, which allowed them to selectively separate oil and water with high efficiency. More importantly, the surface displayed not only good mechanical stability but also chemical stability in corrosive liquids owing to the intrinsic properties of the patina and hydrophobic coating. Furthermore, the surface can be utilized as coating material for the decoration of building exteriors and prevention from surface fouling. We believe that our proposed method would make it possible to develop engineering materials that require robust anti-fouling, anti-frost, and anti-corrosion properties in marine environments.
\end{abstract}

\section{Introduction}

Special wetting surfaces inspired by natural materials have attracted great interest over the past decade because of their potential for practical applications. ${ }^{1-3}$ In particular, a number of methods that can be used to prepare special wetting surfaces with superhydrophilicity or superhydrophobicity have been introduced to make use of their unique functionalities. Superhydrophilic surfaces, which are generally defined by a very low water contact angle (less than $10^{\circ}$ ) and permit complete spreading of a water drop within a few seconds, have been used on antifogging surfaces, ${ }^{4-6}$ in bio-fouling devices ${ }^{7,8}$ and for enhanced boiling heat transfer. ${ }^{9,10}$ On the other hand, superhydrophobic surfaces, which are characterized by a very high water contact angle (greater than $150^{\circ}$ ) and a low contact-angle hysteresis (less than $10^{\circ}$ ), have been used with self-cleaning paints, ${ }^{11-13}$

\footnotetext{
${ }^{a}$ Department of Mechanical Engineering, Pohang University of Science and Technology, Pohang, Gyeongbuk, 790-784, Republic of Korea. E-mail: whwang@postech.ac.kr; Fax: +82-54-279-5899; Tel: +82-54-279-2174

${ }^{b}$ School of Mechanical Engineering, Chung-Ang University, Seoul, 156-756, Republic of Korea. E-mail: slee98@cau.ac.kr; Fax: +82-2-814-9476; Tel: $+82-2-820-5071$

$\dagger$ Electronic supplementary information (ESI) available: The composition of the plating bath and process parameters for electroplating copper film on a stainless steel mesh; FE-SEM images of the fabricated mesh covered with brochantite structure and copper thiolate structure; FE-SEM images and XRD patterns of CuO structure; movie about separation of oil and water using a superhydrophilic mesh; movie about superhydrophobic artificial lotus leaves. See DOI: 10.1039/c4cp05590j
}

as anti-corrosion coatings, ${ }^{14-16}$ in anti-icing functions for heat exchangers, ${ }^{17-20}$ and for drag reduction functions of fluidic systems. ${ }^{21-23}$ Recently, another class of special wetting materials designed by combining superhydrophilicity with superoleophobicity, or super-hydrophobicity with superoleophilicity, has generated considerable attention because these materials make it possible to selectively separate a mixture of oil and water simply through their different interfacial effects, without the addition of any special functions. ${ }^{24-26}$ These special wetting materials have been developed in various ways. Zhang et al. reported the fabrication of $\mathrm{Cu}(\mathrm{OH})_{2}$ nanowire-haired superhydrophilic membranes that allowed effective separation of oil and water. ${ }^{27}$ Gao et al. fabricated $\mathrm{TiO}_{2}$-based meshes with super-hydrophobicity via coatings of self-assembled monolayers (SAMs). ${ }^{28}$ However, it was found that $\mathrm{Cu}(\mathrm{OH})_{2}$ could be easily transformed into more stable copper products, such as $\mathrm{CuO}$ and $\mathrm{Cu}_{2} \mathrm{O}$, so that their surface morphology and the wetting properties could be changed. ${ }^{29}$ Although adding a SAM coating on a rough structure is a common method to fabricate a superhydrophobic surface, the resulting surface exhibits limited stability and moleculesized defects in a corrosive medium, ${ }^{30,31}$ which could degrade the wetting properties. In particular, the development of materials that might be used in seawater for the removal of oil pollution should take into account the surface's corrosionresistant property. Hence, the surface can be strengthened through the incorporation of corrosion-resistant materials and a robust superhydrophobic film for long-term use with good performance in marine applications. 
Copper has good corrosion resistance as well as excellent mechanical and electrical properties so that it is widely used as a basic material in various industrial fields. When copper is exposed to a moist atmosphere or marine environment over long periods, green corrosion products known as patinas, such as those found on the Statue of Liberty, are developed on the surface. The patina commonly consists of two different layers: an inner layer of cuprite $\left(\mathrm{Cu}_{2} \mathrm{O}\right)$ and an outer layer of brochantite $\left(\mathrm{Cu}_{4} \mathrm{SO}_{4}(\mathrm{OH})_{6}\right) \cdot{ }^{32,33}$ Owing to the low corrosion rate and good adhesion strength of the brochantite, patina plays an important role in protecting the underlying metal from further corrosion. ${ }^{34-36}$ As the natural process for the formation of patina on a copper surface takes many years, many methods have been developed to produce it artificially by accelerating the process. ${ }^{37-39}$ However, these methods have problems in attaining uniform brochantite structures on the copper surface and the artificially induced patinas tend to show poor adhesion so that post-process treatments are sometimes required for improving adhesion of the patina to the metal surface. ${ }^{40,41}$ As a result, the application of patina has been limited despite its advantages. Therefore, it is necessary to develop a new strategy for achieving uniform and robust brochantite rapidly as a sustainable corrosion-resistant material even in seawater.

In this work, we report rapid fabrication of robust, superhydrophilic or superhydrophobic, green patina surfaces over large areas and on various copper-coated substances. The green patina surfaces were prepared by two-step oxidization involving the formation of a base oxide on copper and changing of the oxide into a secondary product, i.e., brochantite. The fabrication of the patina surfaces were optimized by considering both brochantite formation and changes in the surface wettability during treatments. The color of the surfaces gradually changed with increasing reaction time, and color-tuned surfaces could be easily obtained by controlling the duration of the reaction. We also demonstrated that the wettability of the patina surface could be converted from superhydrophilic to superhydrophobic by chemical modification. The as-prepared surfaces exhibited superhydrophilicity with underwater superoleophobicity or superoleophilicity with under-oil superhydrophobicity, which allowed them to selectively separate oil and water with high efficiency. More importantly, owing to the intrinsic properties of the patina and hydrophobic coating, the surfaces displayed not only good mechanical stability in adhesion tests but also chemical stability in corrosive liquids. These advances would enable us to develop engineering materials for maritime environments as well as aesthetic embellishments of artworks and architectural structures.

\section{Experimental section}

\subsection{Materials}

Copper foils (99.9\% purity, $0.675 \mathrm{~mm}$ thickness) were supplied by Alfa Aesar, UK. Potassium chlorate $\left(\mathrm{KClO}_{3}\right)$, copper(II) sulfate pentahydrate $\left(\mathrm{CuSO}_{4} \cdot 5 \mathrm{H}_{2} \mathrm{O}\right)$, and 1-dodecanethiol were purchased from Sigma-Aldrich, USA. All other chemicals were purchased from Samchun Chemical, Korea.

\subsection{Fabrication of superhydrophilic/phobic green copper patina}

The copper foils were immersed in $30 \mathrm{wt} \%$ nitric acid for $30 \mathrm{~s}$ to remove surface oxide and then ultrasonically cleaned in ethanol and deionized water for $5 \mathrm{~min}$. The cleaned copper foils were first oxidized using a $0.02 \mathrm{M} \mathrm{KClO}_{3}$ solution at $60{ }^{\circ} \mathrm{C}$ for $12 \mathrm{~h}$. The $\mathrm{pH}$ of the solution was adjusted to 3 by the addition of sulfuric acid. When this initial reaction was completed, $0.1 \mathrm{M} \mathrm{CuSO}_{4} \cdot 5 \mathrm{H}_{2} \mathrm{O}$ was added to the solution with stirring. After a certain amount of time for the reaction, a green patina film was established on the surface. The copper foils were removed and washed with deionized water. In order to add superhydrophobic properties to the as-prepared patina film, the surface was chemically modified using a $10 \mathrm{mM}$ ethanol solution of 1-dodecanethiol at room temperature and then washed with ethanol.

\subsection{Growth of green copper patina film on stainless steel mesh}

A copper film was electrochemically deposited on a cleaned stainless steel mesh with a mesh number of 300 (the average pore diameter was approximately $50 \mu \mathrm{m}$ ). To improve the adhesion between the copper film and stainless steel, Wood's nickel strike process was applied before copper plating. The composition of the plating bath and the process parameters are listed in ESI, $\dagger$ Table S1. The as-prepared copper-plated mesh was washed with deionized water and dried in an oven. A green patina film was then grown on the mesh using the same chemical procedures described above.

\subsection{Instrumentation and characterization}

Scanning electron microscope (SEM) images were obtained to investigate the surface morphology by using a field-emission SEM (FE-SEM; JSM-7401F, JEOL). X-ray diffraction (XRD) was carried out using a D/MAX-2500 (Rigaku) diffractometer with $\mathrm{Cu}-K_{\alpha}$ radiation $(40 \mathrm{kV}, 100 \mathrm{~mA})$. The $\mathrm{XRD}$ data were recorded within the range $2 \theta=10-80^{\circ}$ at a scanning speed of $4^{\circ} \mathrm{min}^{-1}$. The static contact angle and sliding angle on the patina surface were measured using a liquid-droplet analysis tool (SmartDrop, Femtofab Co., Ltd, Korea). The size of the liquid droplet was $5 \mu \mathrm{L}$, and the chosen liquids for the measurements included deionized water, 1,2-dichloroethane, gasoline, diesel, crude oil, $n$-hexadecane, and benzene. All contact-angle results were averaged over five measurements on different areas of each specimen at room temperature. The experiment for separating oil and water was performed using the superhydrophilic or superhydrophobic mesh, which was fixed between a glass funnel and a support with a diameter of $23 \mathrm{~mm}$. The oil-water mixtures $(20 \mathrm{v} / \mathrm{v} \%)$ were poured onto the mesh and the separation was achieved by gravity. After separation, the oil concentration in the collected water was measured using a UV fluorometer oil analyzer (TD-3100, Turner Designs Hydrocarbon Instruments, Inc., USA), and anhydrous hexane was used to extract the oil from the water. The durability of the green patina surface was estimated by measuring changes in the static contact angles with time after immersing the samples in a $0.6 \mathrm{M} \mathrm{NaCl}$ solution and a $0.5 \mathrm{M}$ 
$\mathrm{Na}_{2} \mathrm{SO}_{4}$ solution. A tape peeling test was conducted to assess the adhesion of the green patina superhydrophilic/phobic surfaces in accordance with ASTM D3359-09. A cutting tool with 11 teeth, each separated from the next by $1.0 \mathrm{~mm}$, was used to make the cross-hatch pattern. To ensure good contact between the tape and patina surface, $3 \mathrm{M} \# 610$ scotch tape was applied over the cut, rubbed firmly with an eraser, and then removed. Indentation tests were performed using a nanoindenter (Nano Indenter $\mathrm{XP}^{\mathrm{TM}}$, MTS System, USA) with a Berkovich (three-sided pyramidal) diamond indenter tip. Indentation was performed at approximately $10 \mathrm{~m} \mathrm{~s}^{-1}$ to a depth of $2 \mu \mathrm{m}$ with a hold period of $10 \mathrm{~s}$ at the peak load.

\section{Results and discussion}

The superhydrophilic/phobic green patina surfaces were fabricated by two-step chemical oxidation of a copper surface and chemical modification. The cleaned copper plates were first immersed in the acidic oxidizing solution to form a base oxide film. After the addition of copper sulfate to the solution, the prepared oxide film transformed into green patina during the reaction period (12-72 h). FE-SEM measurements revealed the morphological developments in the as-prepared samples. Fig. 1a shows the copper oxide layer with cubic crystal structures after initial stages of the reaction. After a reaction duration of $12 \mathrm{~h}$, dandelion-like microstructures with dimensions of over $10 \mu \mathrm{m}$ were grown loosely on the surface, but they became more compact during latter stages of the reaction, as shown in Fig. 1b-d. Moreover, the number of crystalline structures markedly increased and the surface was completely covered with the structures after $60 \mathrm{~h}$ of the treatment. As the number of structures increased, it was observed that the surface changed from a reddish to heavy greenish color (see insets of Fig. 1). The formation of the green patina was confirmed by XRD. Fig. 1e shows the XRD spectra of as-prepared patina samples, which were normalized against the maximum intensity. The diffraction peaks of the reddish copper oxide layer match the powder diffraction file of $\mathrm{Cu}_{2} \mathrm{O}$, namely cuprite (PDF \# 05-0667). In contrast, after the addition of copper sulfate, a set of new peaks appeared, corresponding to that of monoclinic $\mathrm{Cu}_{4} \mathrm{SO}_{4}(\mathrm{OH})_{6}$, brochantite (PDF \# 43-01458), except for the peaks (marked with $\star$ ) from the copper substrate. A comparison of the XRD results with results of previous studies show that the green patina layer was composed of brochantite and an inner layer of cuprite. ${ }^{42}$ The peak intensities of copper and cuprite decreased when compared with those of brochantite during the treatment because the thick brochantite layer caused a significant decrease in the XRD intensities. After a reaction duration of $60 \mathrm{~h}$, there were no significant changes in the XRD intensities among copper, cuprite, and brochantite, indicating that the sample surface was completely covered with brochantite.

The green patina growth is essentially a double oxidation process of the copper surface, as illustrated in Fig. 1f. In an aqueous acidic solution, the surface of copper is rapidly oxidized by $\mathrm{KClO}_{3}$, the oxidizing agent, and the cuprite is formed as follows: ${ }^{43}$

$$
6 \mathrm{Cu}+\mathrm{ClO}_{3}^{-} \rightarrow 3 \mathrm{Cu}_{2} \mathrm{O}+\mathrm{Cl}^{-}
$$

Brochantite is then produced by the reaction between $\mathrm{Cu}^{2+}$ ions from oxidation of the cuprite and $\mathrm{SO}_{4}{ }^{2-}$ ions added to the solution, as shown in the following reaction: ${ }^{33,44}$

$$
2 \mathrm{Cu}_{2} \mathrm{O}+\mathrm{SO}_{4}^{2-}+4 \mathrm{H}_{2} \mathrm{O} \rightarrow \mathrm{Cu}_{4} \mathrm{SO}_{4}(\mathrm{OH})_{6}+2 \mathrm{H}^{+}+4 \mathrm{e}^{-}
$$
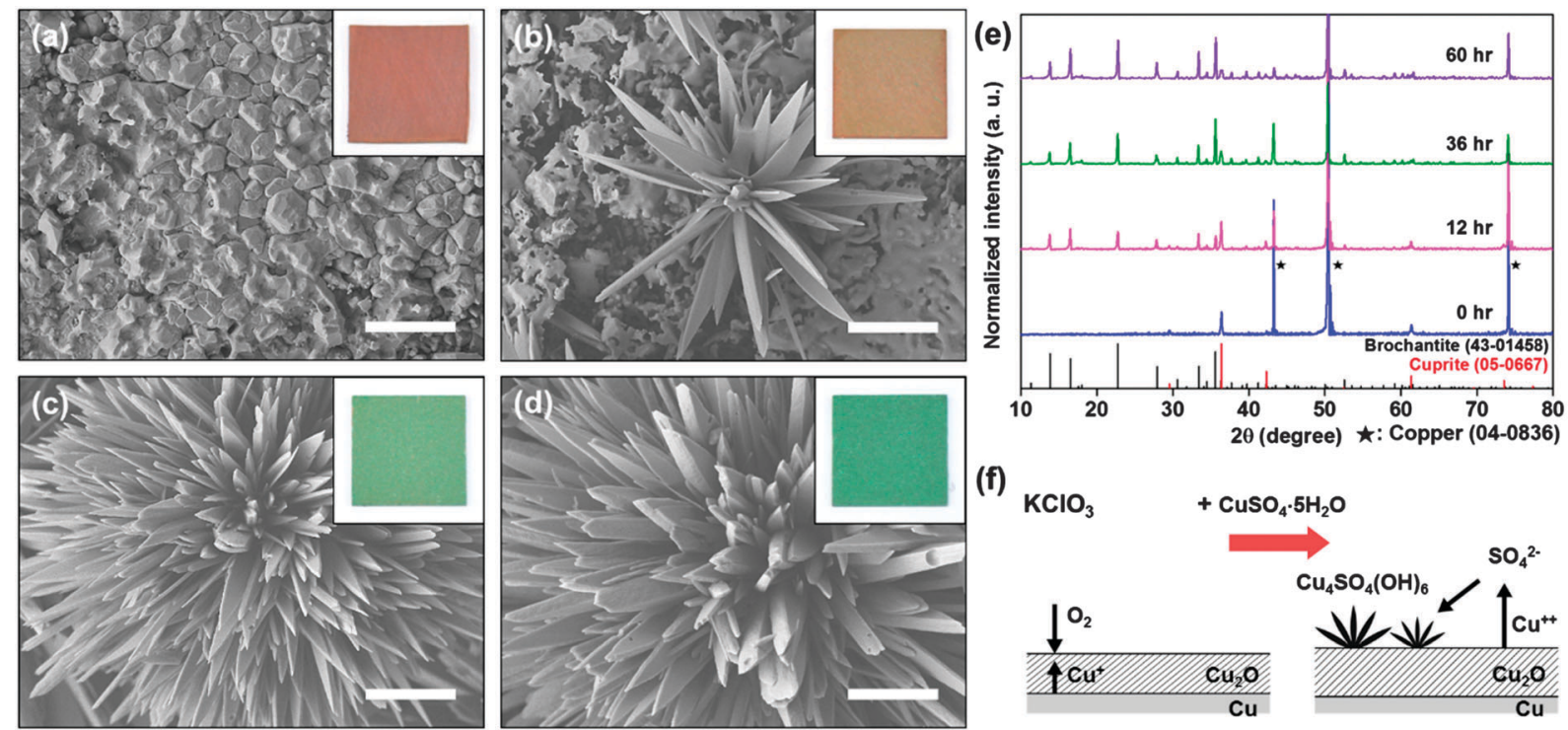

(f)
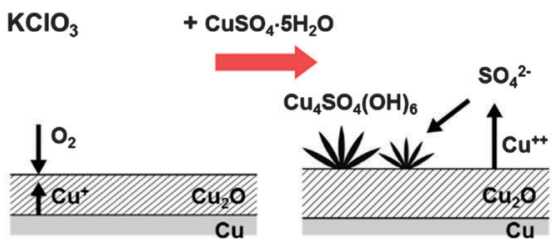

Fig. 1 FE-SEM images and XRD patterns of the prepared sample surfaces. (a) Initial copper oxide layer. (b-d) Development of the dandelion-like microstructure after different reaction durations of copper sulfate in an oxidizing solution: (b) 12 h; (c) 36 h; (d) 60 h. The insets show the sample surface changing from a reddish to heavy green color. All scale bars correspond to a length of $5 \mu \mathrm{m}$. (e) XRD patterns of the cuprite and brochantite film grown on a copper plate. The solid lines indicate the XRD patterns of cuprite (PDF \#05-0667) and brochantite (PDF \#43-01458) from the powder diffraction database. The symbols $\star$ represent peaks of the copper plate. (f) Schematic illustration of the brochantite formation. 
However, the cuprite is continuously dissolved by $\mathrm{H}^{+}$and $\mathrm{ClO}_{3}{ }^{-}$ ions, as shown in eqn (3) and (4). The presence of $\mathrm{Cu}^{2+}$ ions in the solution is required to prevent the cuprite from dissolving excessively. Therefore, the addition of copper sulfate provides a stable base oxide as well as the composition of brochantite.

$$
\begin{gathered}
\mathrm{Cu}_{2} \mathrm{O}+2 \mathrm{H}^{+} \rightarrow \mathrm{Cu}+\mathrm{Cu}^{2+}+\mathrm{H}_{2} \mathrm{O} \\
3 \mathrm{Cu}_{2} \mathrm{O}+\mathrm{ClO}_{3}^{-}+12 \mathrm{H}^{+} \rightarrow 6 \mathrm{H}_{2} \mathrm{O}+6 \mathrm{Cu}^{2+}+\mathrm{Cl}^{-}
\end{gathered}
$$

To study the wetting properties of the as-prepared samples, the water contact angle (WCA) in air, the oil contact angle (OCA), and the underwater oil sliding angle (OSA) were examined in detail. Fig. 2a represents the variations in contact angles with the formation of green patina. In the early stage of the reaction, the sample exhibited slight hydrophobicity with a measured WCA of $91.9^{\circ}$. As the reaction time increased, the sample became hydrophilic and the water droplet was eventually spread over the sample surface after $24 \mathrm{~h}$ of reaction. This wetting behavior implies that the surface roughness increased owing to the growth of the crystalline structures, and the drastic changes in WCA can be attributed to the capillary effect related to the dandelionlike microstructures. The microstructures with large roughness amplify the wettability of the surface in accordance with
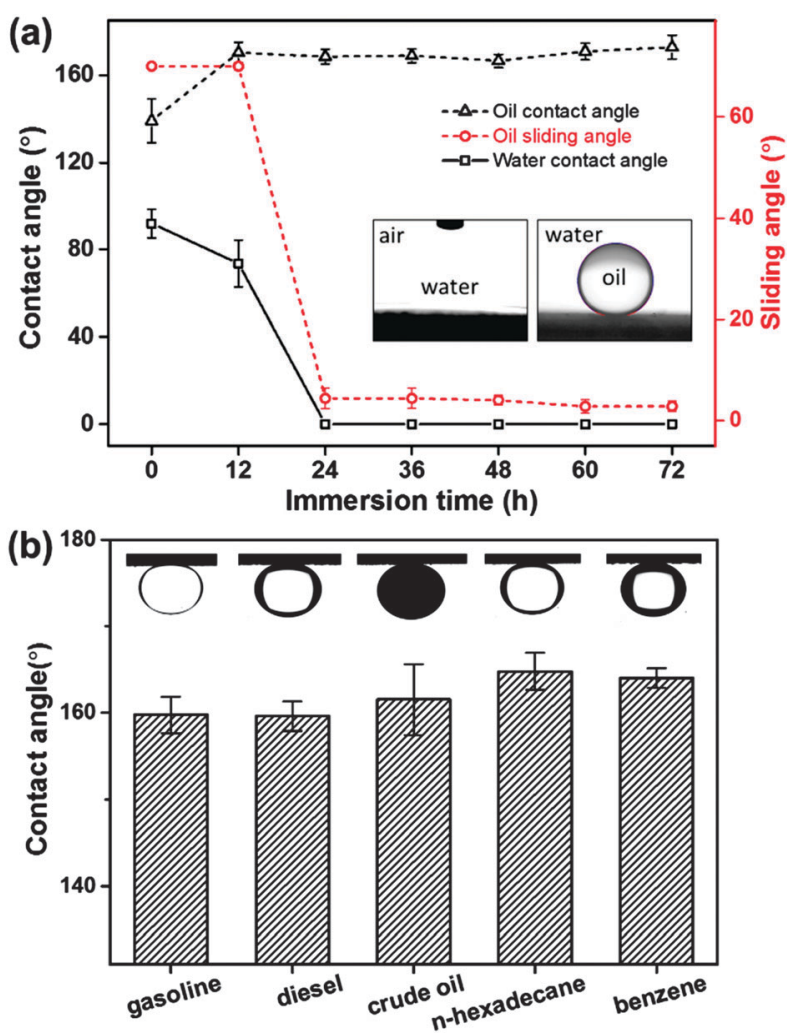

Fig. 2 (a) Changes in water and oil contact angles with reaction time. As the reaction time increased, the hydrophilicity increased and the corresponding underwater oleophobicity also increased. The as-prepared sample showed superhydrophilicity and underwater superoleophobicity after $24 \mathrm{~h}$ of the treatment. (b) Contact angles of various organic oils on the green patina samples (60 h of reaction time) that were secured horizontally upside down. The volume of each oil droplet was about $5 \mu \mathrm{L}$.
Wenzel's equation ${ }^{45}$ and waterdroplets quickly spread out as soon as they come in contact with the surface. Moreover, as the reaction time increased, the reverse trend was observed in the variations in OCA of various organic oils on different samples. As the reaction time exceeded $24 \mathrm{~h}$, the samples exhibited underwater superoleophobicity with a high OCA close to $170^{\circ}$ and OSA below $5^{\circ}$. Compared to the patina's superhydrophilicity in air, the underwater superoleophobicity was achieved by trapped water inside the microstructure's cavities resulting from the roughened surface because this trapped water reduced the actual contact area between the solid surface and oil droplet. The green patina surfaces also showed such special wetting properties for organic oils including gasoline, diesel, crude oil, $n$-hexadecane, and benzene. To obtain the OCA measurements, the samples were immersed in a cuvette filled with water and trapped air bubbles on the surface were removed using ultrasonic equipment. With the samples secured horizontally upside down, various oils were introduced into the solid-water system using a curved needle. As shown in Fig. 2b, when oil drops were introduced, the OCA ranged from $160^{\circ}$ for gasoline to $164^{\circ}$ for hexadecane, and the sliding angles for all examined liquids were below $10^{\circ}$.

For the conversion of their wetting properties from superhydrophilicity to superhydrophobicity, the as-prepared green patina samples were immersed in a $10 \mathrm{mM}$ ethanol solution of 1-dodecanethiol for $10 \mathrm{~min}$ to $24 \mathrm{~h}$. Previous studies have shown the preparation of superhydrophobic surfaces through the reaction between $\mathrm{Cu}(\mathrm{OH})_{2}$ and alkyl thiols. ${ }^{46,47}$ Using a similar method, brochantite reacted with 1-dodecanethiol to form copper thiolates, as shown in the following equation:

$$
\mathrm{Cu}_{4} \mathrm{SO}_{4}(\mathrm{OH})_{6}+6 \mathrm{C}_{n} \mathrm{H}_{2 n+1} \mathrm{SH} \rightarrow \mathrm{Cu}_{4} \mathrm{SO}_{4}\left(\mathrm{SC}_{n} \mathrm{H}_{2 n+1}\right)_{6}+6 \mathrm{H}_{2} \mathrm{O}
$$

The modifying reactant 1-dodecanethiol is composed of a mercapto group (-SH) and long-chain alkyl groups $\left(\mathrm{C}_{n} \mathrm{H}_{2 n+1}{ }^{-}\right)$ with low surface energy. The mercapto group could be coordinated with copper ions and etch the microstructured brochantite, yielding morphological changes as a result. The FE-SEM images in Fig. 3a-d show the changes in surface morphology after a sample was immersed in the solution for different periods of time. As the immersion period increased, the dandelion-like structures became shorter and blunter. In addition, the simple immersion in the ethanol solution of 1-dodecanethiol induced the color change from green to yellow-green over the entire sample surface (see insets of Fig. 3). It is known that the color of a surface stems from the interference between the reflected light from the air-film interface and the film-substrate interface. ${ }^{48,49}$ As the reflected light intensity is influenced by the film thickness, the color variation on the sample surfaces could be attributed to decreasing film thickness resulting from the thiolate reaction. Further studies are needed to understand the relationship between the thickness of the green patina and its surface color, however. Meanwhile, the copper thiolates anchored on the surface brought about changes in the wetting behavior from superhydrophilicity to superhydrophobicity. Fig. 3e exhibits the wetting properties of water and oil on the samples that were modified by 1-dodecanethiol. The as-prepared samples showed 

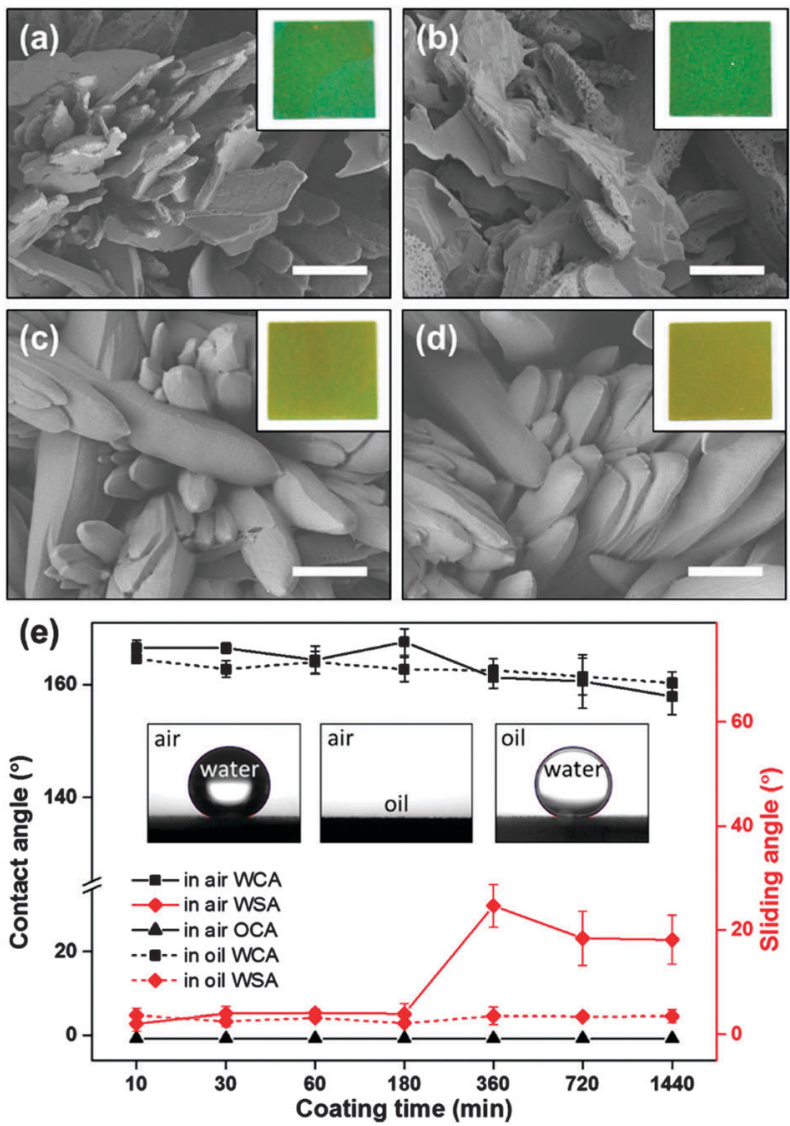

Fig. 3 FE-SEM images of the green patina sample after treatment with $10 \mathrm{mmol}$ ethanol solution of 1-dodecanethiol for (a) $30 \mathrm{~min}$, (b) $1 \mathrm{~h}$, (c) $3 \mathrm{~h}$, and (d) $6 \mathrm{~h}$. The green patina film was formed after $60 \mathrm{~h}$ of the reaction. The insets show that the color of the sample surface gradually turned yellow. All scale bars correspond to a length of $5 \mu \mathrm{m}$. (e) Water and oil contact angles of green patina samples modified with 1-dodecanethiol. The samples exhibited superhydrophobicity and superoleophilicity in air. After over $3 \mathrm{~h}$ of modification, the value of WSA increased despite a high WCA. In oil, however, the samples had a high WCA of $162.6 \pm 1.45^{\circ}$ and very low WSA of $3.07 \pm 0.55^{\circ}$, showing improved super-hydrophobicity.

superhydrophobicity and superoleophilicity in air. For the samples treated with a relatively short immersion time $(\sim 3 \mathrm{~h})$, a high WCA of $166.3 \pm 1.35^{\circ}$ and a low water sliding angle (WSA) of $3.45 \pm 0.98^{\circ}$ were observed. According to the Cassie and Baxter equation, a high area fraction of air decreases greatly the solid/water interface and prevents penetration of water into the surface. ${ }^{50}$ Thus, it can be inferred that the microstructured surface plays an important role in the enhancement of hydrophobicity. With longer modification time, the measured value of WSA was abruptly increased despite the high value of WCA. This phenomenon of sticky water droplets resulted from a decrease in the surface roughness owing to the thiolate reaction. However, when the samples were submerged in oil, it showed under-oil superhydrophobicity and the water droplets easily rolled off the surface. In oil, owing to their superoleophilicity, the sample surfaces were easily infused with oil. Similar to the superhydrophilic surface, the trapped oil within the sample surface served as a repulsive liquid and greatly reduced the water-solid contact area. Consequently, the decrease in the surface roughness had relatively less impact on the wetting property, and the under-oil superhydrophobicity was improved.

Based on our previous results, the green patina surfaces could be engineered for robust materials to selectively separate oil and water mixtures. To study this function, meshes were fabricated by two-step oxidation of the copper-plated stainless steel mesh and surface modification, after which brochantite and copper thiolates structures uniformly covered the copperplated steel mesh (see ESI, $\dagger$ Fig. S1). These structures captured water (or oil) and formed an oil-water-solid interface, which is a key factor for separating oil and water. As shown in Fig. 4a and ESI, $\dagger$ Movie S1, water rapidly passed through the superhydrophilic mesh and collected in the beaker after the mixture of oil and water was poured over the as-prepared mesh. However, the density of the used oil was lighter than water, as was the case for the superhydrophobic mesh, and the oil stayed above the water once the water flowed into the mesh ahead of the oil, as shown in Fig. 4b. As a result, the upper oil and water layer had to be removed using a pipette and separated once again to completely isolate the oil from water. The separation efficiency was calculated using the following equation: ${ }^{24}$

$$
\text { Separation efficiency }(\%)=\left(1-\frac{C_{\text {after }}}{C_{\text {before }}}\right) \times 100
$$

where $C_{\text {before }}$ is the measured oil concentration of the mixture before the separation test and $C_{\text {after }}$ is the measured oil concentration of the separated water after the test. Fig. 4c shows that the separation efficiencies of the superhydrophilic mesh were as high as $99 \%$ and almost all (except for the crude oil) of the oils were successfully removed. The low separation efficiency for the crude oil was thought to be caused by the rheological properties and complex composition of the crude oil. On the other hand, the separation efficiencies of the superhydrophobic mesh were slightly decreased because a small amount of the oil remained above the water. We could not measure the concentration of the crude oil in water because the walls of the device were severely fouled by crude oil. However, these problems could be alleviated by using an inclined superhydrophobic mesh that is in contact with both water and oil simultaneously or by installing water drainage to prevent secondary pollution.

The corrosion resistance of the green patina surfaces in marine environments was investigated by immersing the samples in aqueous corrosive solutions. Fig. 5a shows the variations in the contact angle on the superhydrophilic and superhydrophobic sample surfaces after they were immersed in a $0.6 \mathrm{M} \mathrm{NaCl}$ solution for 7 days. For the green patina surfaces with superhydrophilicity, no obvious changes in WCA and underwater OCA were observed, and the surface retained its superhydrophilicity with a WCA of nearly $0^{\circ}$ and its underwater superoleophobicity with an OCA of over $150^{\circ}$ for a week. This high corrosion resistance of the surface could have originated from the nature of the brochantite, the main composition of the green patina. The low corrosion rate of the brochantite is an important factor in the provision of protection against the corrosive solution. ${ }^{35,51}$ Meanwhile, similar results were observed in the superhydrophobic green patina surfaces. 

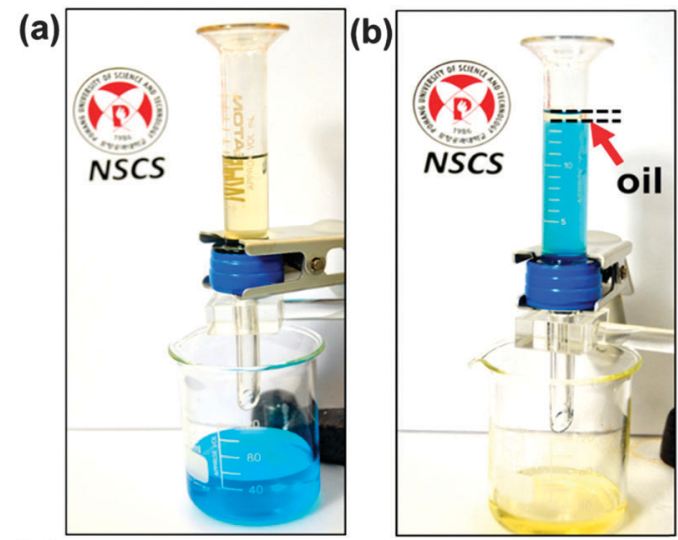

(c)

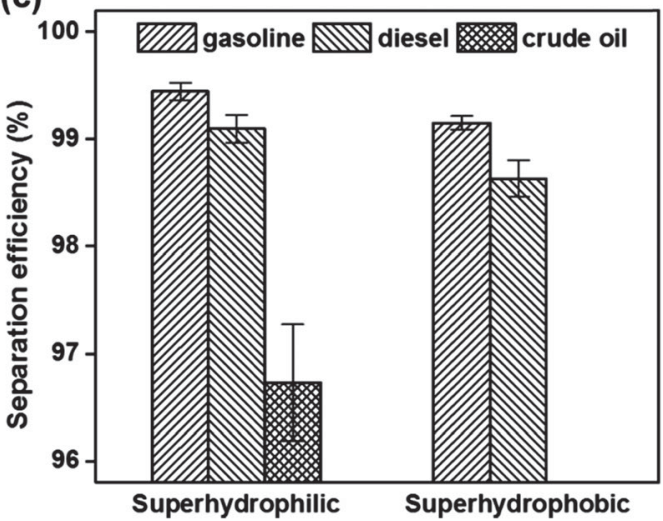

Fig. 4 Experimental apparatus for separating gasoline-water mixtures. The water was dyed blue. (a) Water selectively passed through the superhydrophilic mesh and oil remained in the upper part of the device. Prior to the test, the superhydrophilic mesh was intentionally wetted with water. (b) On the contrary, oil passed through the superhydrophobic mesh while water remained in the upper part of the device. The device became clogged by water and oil permeation was prevented. (c) Separation efficiency of the as-prepared meshes; gasoline, diesel, and crude oil were used in the test.

The samples exhibited superoleophilicity in air and superhydrophobicity under oil after they were removed from the solution. The trapped air at the water-solid interface minimized the contact area and inhibited the penetration of ions such as $\mathrm{Cl}^{-}$. Moreover, the corrosion resistant property could be improved because the thiolate reaction formed a relatively thick hydrophobic polymolecular layer ${ }^{52}$ compared to the self-assembled monolayer coating that was already known to be hydrophobic. It can be seen that our surfaces also retained their wettability by water and oil when immersed in a $0.5 \mathrm{M} \mathrm{Na}_{2} \mathrm{SO}_{4}$ solution, as shown in Fig. 5b. Furthermore, the weight of superhydrophilic/ hydrophobic samples remained almost unchanged until after immersion in the solutions for 7 days. These results show that our superhydrophobic green patina surfaces had superior corrosion resistant property when compared with previously reported surfaces. ${ }^{53}$

To provide further insight into the mechanical stability of our surfaces, an adhesion test was performed in accordance with the procedures described in ASTM D 3359-09e2. For comparison, the hierarchical CuO surface was prepared following chemical oxidation ${ }^{54,55}$ and the surface was also modified
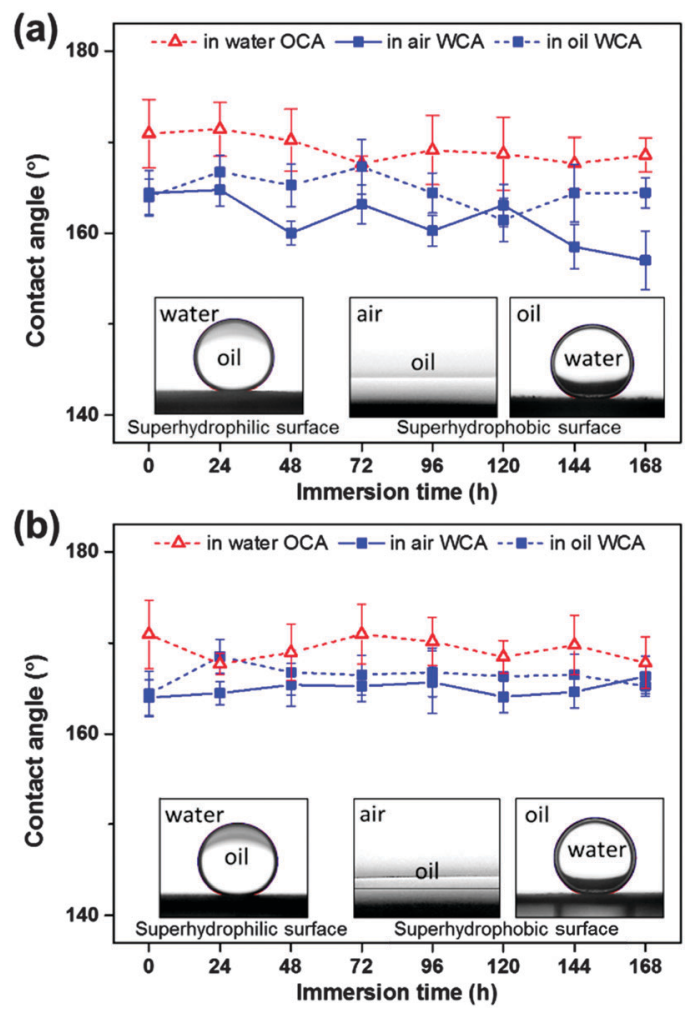

Fig. 5 Changes in contact angles of water and oil after the samples were immersed for 7 days in (a) a $0.6 \mathrm{M} \mathrm{NaCl}$ solution and (b) a $0.5 \mathrm{M} \mathrm{Na}_{2} \mathrm{SO}_{4}$ solution. The superhydrophilic green patina samples were obtained after $60 \mathrm{~h}$ of reaction. The superhydrophobic green patina samples were obtained from modification of superhydrophilic samples with 1-dodecanethiol for $1 \mathrm{~h}$. The insets show droplets on the surfaces of samples removed from the corrosive solutions after 7 days.

with 1-dodecanethiol (see ESI, $\dagger$ Fig. S2). Fig. 6a-d show photographs of the sample surfaces after the cross-cut tape test. The superhydrophilic and superhydrophobic green patina surfaces exhibited excellent adhesion onto the copper substrate, corresponding to the $4 \mathrm{~B}$ to $5 \mathrm{~B}$ classification. And there were no changes in the superhydrophobicity after the test. On the other hand, entire squares on the CuO surface layer were detached after the test. This exfoliation problem can cause deterioration in the superhydrophobicity of the surface. Moreover, the mechanical properties of the fabricated surfaces were measured by using a nanoindentation test. As can be seen in Fig. 6e, the patina surfaces appeared to have superior mechanical properties such as high modulus and surface hardness relative to $\mathrm{CuO}$ surfaces. In the superhydrophilic patina surface, modulus is $7.20 \pm 1.018 \mathrm{GPa}$ and hardness is $65.3 \pm 21.4 \mathrm{MPa}$. The modulus of the CuO surface is $3.26 \pm 0.702 \mathrm{GPa}$ and hardness is $46.9 \pm 5.89 \mathrm{MPa}$. However, the values of superhydrophobic patina surfaces decreased to $2.50 \pm 0.609 \mathrm{GPa}$ and $19.8 \pm 7.55 \mathrm{MPa}$, respectively. Similarly the modulus and hardness of the superhydrophobic $\mathrm{CuO}$ surface were lowered to $1.385 \pm 0.342 \mathrm{GPa}$ and $13.4 \pm 1.670 \mathrm{MPa}$, respectively. Because the surface structures were etched during surface modification with 1-dodecanethiol, the mechanical properties of the superhydrophobic surfaces would be eventually reduced. 

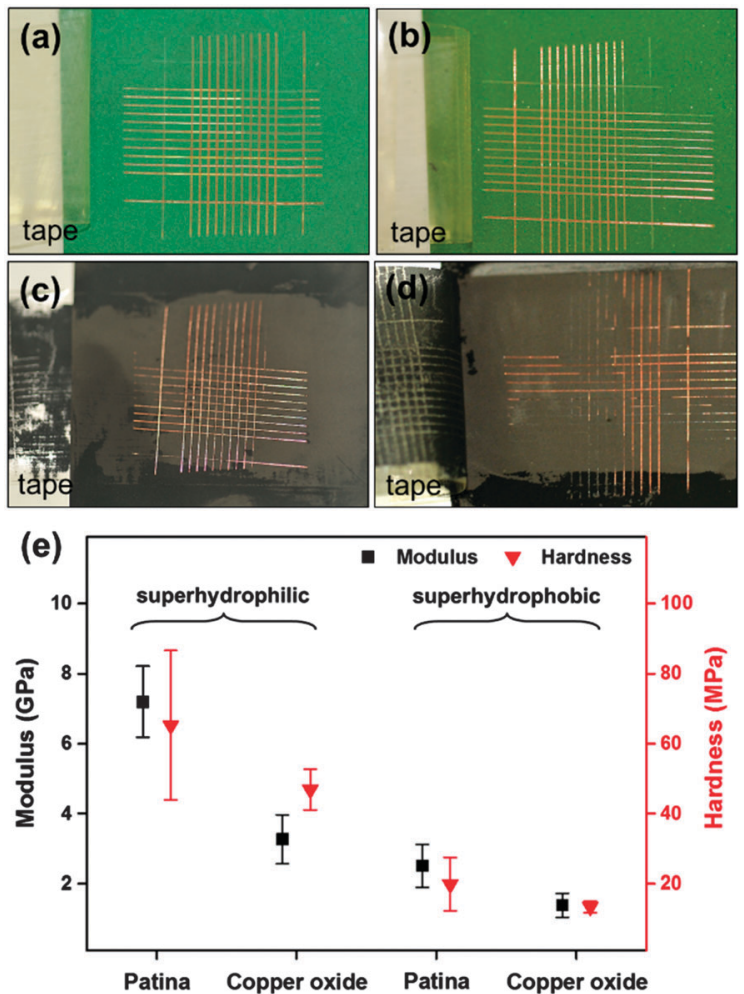

Fig. 6 Photographs of the surfaces after adhesion test using Scotch tape: (a) superhydrophilic green patina surface, (b) superhydrophobic green patina surface, (c) superhydrophilic $\mathrm{CuO}$ surface (d) superhydrophobic $\mathrm{CuO}$ surface prepared by following the procedure described in the references. ${ }^{54,55}$ The superhydrophilic and superhydrophobic green patina surfaces showed excellent adhesion onto the copper substrate, but the $\mathrm{CuO}$ surface exhibited poor adhesion. (e) Indentation modulus and surface hardness of superhydrophilic/ phobic patina surfaces and superhydrophilic/phobic CuO surfaces. The modulus and hardness of the patina surfaces are greater than those of the CuO surface.

Our green patina surfaces could also be used for the surface decoration of architectural structures with anti-fouling and corrosion-resistant properties. As an example of possible application

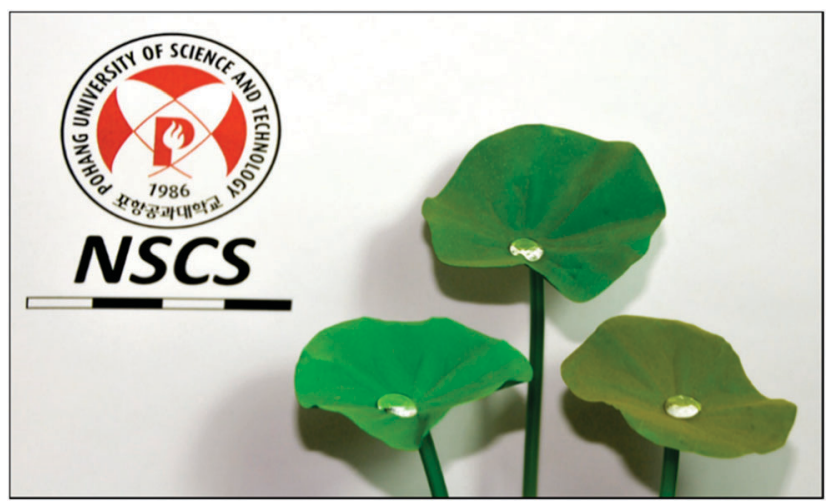

Fig. 7 Photograph of superhydrophobic artificial lotus leaves. The leaves were made of copper plates, and they colored by forming green patina and surface modification. Different colored surfaces could be obtained by controlling the reaction time of brochantite and surface modification. The scale bar corresponds to a length of $10 \mathrm{~cm}$. in artworks, Fig. 7 and ESI, $\dagger$ Movie S2, show artificial superhydrophobic lotus leaves. The leaves were made of copper plates and the green color was produced by oxidation and chemical surface modification. In addition, our fabrication process can be applied to the surfaces with various geometry or shape, which is an important feature for broadening the application of superhydrophilic/phobic green patina surfaces.

\section{Conclusions}

We successfully fabricated robust superhydrophilic or superhydrophobic green patina surfaces on copper substrates using two-step oxidization involving the formation of cuprite and brochantite. The fabricated green patina surfaces exhibited superhydrophilicity in air and superoleophobicity underwater, and the wetting properties could be converted to superoleophilicity in air and superhydrophobicity under oil after chemical modification. The colors of the surface were easily controlled by the process time of brochantite formation and surface modification. The as-prepared surfaces also provided superior corrosion resistance and mechanical durability. We showed that the robust green patina surfaces could be applied for selective separation of oil and water. Furthermore, the surfaces could be utilized not only as aesthetically pleasing materials for the decoration of building exteriors or sculptures, but also as coatings to prevent surface fouling. We believe that our proposed method would be favorable for many applications that require robust anti-fouling, anti-frost, and anti-corrosion properties in marine environments.

\section{Acknowledgements}

This work was supported by the National Research Foundation of Korea (NRF) grant funded by the Korea government (MSIP) (No. NRF-2013R1A2A1A01016911).

\section{Notes and references}

1 L. Feng, S. Li, Y. Li, H. Li, L. Zhang, J. Zhai, Y. Song, B. Liu, L. Jiang and D. Zhu, Adv. Mater., 2002, 14, 1857.

2 T. Sun, L. Feng, X. Gao and L. Jiang, Acc. Chem. Res., 2005, 38, 644.

3 X. J. Feng and L. Jiang, Adv. Mater., 2006, 18, 3063.

4 D. Tahk, T.-I. Kim, H. Yoon, M. Choi, K. Shin and K. Y. Suh, Langmuir, 2010, 26, 2240.

5 W. S. Y. Wong, N. Nasiri, A. L. Rodriguez, D. R. Nisbet and A. Tricoli, J. Mater. Chem. A, 2014, 2, 15575.

6 J.-J. Park, D.-Y. Kim, S. S. Latthe, J.-G. Lee, M. T. Swihart and S. S. Yoon, ACS Appl. Mater. Interfaces, 2013, 5, 6155.

7 A. Tiraferri, Y. Kang, E. P. Giannelis and M. Elimelech, Environ. Sci. Technol., 2012, 46, 11135.

8 S. Liang, Y. Kang, A. Tiraferri, E. P. Giannelis, X. Huang and M. Elimelech, ACS Appl. Mater. Interfaces, 2013, 5, 6694.

9 D. Li, G. S. Wu, W. Wang, Y. D. Wang, D. Liu, D. C. Zhang, Y. F. Chen, G. P. Peterson and R. Yang, Nano Lett., 2012, 12, 3385 . 
10 S. Shin, B. Seok Kim, G. Choi, H. Lee and H. Hee Cho, Appl. Phys. Lett., 2012, 101, 251909.

11 Y.-L. Zhang, J.-N. Wang, Y. He, Y. He, B.-B. Xu, S. Wei and F.-S. Xiao, Langmuir, 2011, 27, 12585.

12 S. S. Latthe, C. Terashima, K. Nakata, M. Sakai and A. Fujishima, J. Mater. Chem. A, 2014, 2, 5548.

13 M. Toma, G. Loget and R. M. Corn, ACS Appl. Mater. Interfaces, 2014, 6, 11110.

14 C.-W. Peng, K.-C. Chang, C.-J. Weng, M.-C. Lai, C.-H. Hsu, S.-C. Hsu, Y.-Y. Hsu, W.-I. Hung, Y. Wei and J.-M. Yeh, Electrochim. Acta, 2013, 95, 192.

15 D. Zang, R. Zhu, C. Wu, X. Yu and Y. Zhang, Scr. Mater., 2013, 69, 614.

16 F. Su and K. Yao, ACS Appl. Mater. Interfaces, 2014, 6, 8762.

17 L. B. Boinovich, A. M. Emelyanenko, V. K. Ivanov and A. S. Pashinin, ACS Appl. Mater. Interfaces, 2013, 5, 2549.

18 Y. Wang, J. Xue, Q. Wang, Q. Chen and J. Ding, ACS Appl. Mater. Interfaces, 2013, 5, 3370.

19 T. Jing, Y. Kim, S. Lee, D. Kim, J. Kim and W. Hwang, Appl. Surf. Sci., 2013, 276, 37.

20 Y. Sohn, D. Kim, S. Lee, M. Yin, J. Y. Song, W. Hwang, S. Park, H. Kim, Y. Ko and I. Han, J. Mater. Chem. A, 2014, 2, 11465.

21 S. Lee, J. H. Kang, S. J. Lee and W. Hwang, Lab Chip, 2009, 9, 2234.

22 C. Lee and C.-J. Kim, Phys. Rev. Lett., 2011, 106, 014502.

23 S. Lyu, D. C. Nguyen, D. Kim, W. Hwang and B. Yoon, Appl. Surf. Sci., 2013, 286, 206.

24 Z. Xue, S. Wang, L. Lin, L. Chen, M. Liu, L. Feng and L. Jiang, Adv. Mater., 2011, 23, 4270.

25 J. Zhang and S. Seeger, Adv. Funct. Mater., 2011, 21, 4699.

26 L. Zhang, Y. Zhong, D. Cha and P. Wang, Sci. Rep., 2013, 3, 2326.

27 F. Zhang, W. B. Zhang, Z. Shi, D. Wang, J. Jin and L. Jiang, Adv. Mater., 2013, 25, 4192.

28 C. Gao, Z. Sun, K. Li, Y. Chen, Y. Cao, S. Zhang and L. Feng, Energy Environ. Sci., 2013, 6, 1147.

29 A. Chaudhary and H. C. Barshilia, J. Phys. Chem. C, 2011, 115, 18213.

30 F. Zhang, L. Zhao, H. Chen, S. Xu, D. G. Evans and X. Duan, Angew. Chem., Int. Ed., 2008, 47, 2466.

31 P. Wang, D. Zhang, R. Qiu and J. Wu, Corros. Sci., 2014, 83, 317.

32 P. E. Philip and A. Schweitzer, Corrosion and Corrosion Protection Handbook, CRC Press, 2nd edn, 1988.
33 K. P. Fitzgerald, J. Nairn and A. Atrens, Corros. Sci., 1998, 40, 2029.

34 A. Atrens, Adv. Eng. Mater., 2004, 6, 83.

35 G. Song and A. Atrens, Adv. Eng. Mater., 2007, 9, 177.

36 T. E. Graedel, Corros. Sci., 1987, 27, 741.

37 S. K. Coburn, Atmospheric Factors Affecting the Corrosion of Engineering Metals, ASTM International, 1978.

38 European Commission Directorate-General XII, Science, Research, and Development, Development of a new nondestructive method for analysis of the atmospheric corrosion and corrosion protection of copper and copper alloys: protection and conservation of the European cultural heritage, Office for Official Publications of the European Communities, 1999.

39 B. M. Rosales, R. M. Vera and J. P. Hidalgo, Corros. Sci., 2010, 52, 3212.

40 H. Otmačić Ćurković, T. Kosec, K. Marušić and A. Legat, Electrochim. Acta, 2012, 83, 28.

41 O. Papadopoulou, M. Delagrammatikas, P. Vassiliou, S. Grassini, E. Angelini and V. Gouda, Surf. Interface Anal., 2014, 46, 771.

42 B. Rosales, R. Vera and G. Moriena, Corros. Sci., 1999, 41, 625.

43 S. R. Yoon, PhD thesis, University of Utah, 1971, vol. 8.

44 K. P. Fitzgerald, J. Nairn, G. Skennerton and A. Atrens, Corros. Sci., 2006, 48, 2480.

45 D. Quéré, Annu. Rev. Mater. Res., 2008, 38, 71.

46 X. Wu and G. Shi, J. Phys. Chem. B, 2006, 110, 11247.

47 Z. Cheng, M. Du, H. Lai, N. Zhang and K. Sun, Nanoscale, 2013, 5, 2776.

48 X. Zhao, G. Meng, Q. Xu, F. Han and Q. Huang, Adv. Mater., 2010, 22, 2637.

49 T. Ishizaki and M. Sakamoto, Langmuir, 2011, 27, 2375.

50 S. Wang, L. Feng and L. Jiang, Adv. Mater., 2006, 18, 767.

51 F. Noli, P. Misaelides, A. Hatzidimitriou, E. Pavlidou and M. Kokkoris, J. Mater. Chem., 2003, 13, 114.

52 F. Zucchi, V. Grassi, A. Frignani and G. Trabanelli, Corros. Sci., 2004, 46, 2853.

53 T. Ishizaki, Y. Masuda and M. Sakamoto, Langmuir, 2011, 27,4780 .

54 H. Hou, Y. Xie and Q. Li, Cryst. Growth Des., 2004, 5, 201.

55 D. Zang, C. Wu, R. Zhu, W. Zhang, X. Yu and Y. Zhang, Chem. Commun., 2013, 49, 8410. 